\title{
Dysbiosis, gut, cognitive movement disorders and nonmotor skin diseases.
}

\author{
Chan KTM* \\ Specialist in Dermatology, Hong Kong Academy of Medicine, Hong Kong.
}

\begin{abstract}
Chronic skin eruption like severe scarring Acne vulgaris, Rosacea and Chronic Atopic Dermatitis have a deeper than skin connection to diseases of our internal organs. The relationship of small gut dysbiosis due to gut immobility, stasis, breakdown and atrophy of the intestinal linings and gastrointestinal reflux enhance inflammatory cytokines, toxins, trace elements reaching the brain, skin and other organs of the body. With advances of modern science and technology, Central Nervous System (CNS) neurological disorders like Parkinson disease (PD), Chronic depression and Autism may be intrigued in this complex brain-CNS-Gut-Enteric microbiota - Skin axis jig saw puzzle.
\end{abstract}

Keywords: Gut dysbiosis, Cognitive Movement disorders, Enteric nervous system, Skin.

Accepted on April 05, 2018

\section{Introduction}

The Gastrointestinal (GI) tract especially small intestinal epithelial barrier defects; which primarily function as an important first line physiological, immune and endocrine absorption organ may become aberrant as a result to genetic predisposition and western life style dietary habit. The breaking down of the epithelial cells tight junctions allows diffusions of toxins released from a modern western diet mainly composed of a high content of processed carbohydrate: high sugar and fats ratio relative to low fibres contents, pollutants like heavy metals, preservatives, pesticides, infections, prolonged antibiotics intake, with a low $\mathrm{pH}$ environment into the body circulation. This loss of epithelial cells cohesion of small bowel termed as " leaky gut" has immense consequences to the understanding and pathogenesis of skin disease like rosacea, acne vulgaris and atopic dermatitis; rheumatological disease like rheumatoid arthritis, endocrine diseases like thyroid diseases and adrenal impairments, large bowel disease like inflammatory bowel diseases(IBD), oral cavity and sinus disease like hay fever and interestingly to some of our brain cognitive disorders like depression, autism and Parkinson disease. Though exact detailed pathogenetic pathways, signalling and control mechanisms is still not fully elucidated; factors like malabsorption, autoimmunity with autoantibodies formation to specific organs, inflammation and breaching of the blood brain barrier allowing toxins, cytokines and other signals breach through the blood brain barrier allowing deliverance into our CNS are strongly suggestive (Figures 1 and 2).

\section{Acneiform eruption like severe scaring acne and Rosacea}

A Gut-Brain -Skin hypothesis has been established in acneiform facial skin eruption [1-3]. In severe acne, risk factors like positive family history involving expression of Insulin Growth factor -1 genes (IGF-1) and Androgen Receptor genes; high calorie and high glycaemic index diet consisting of dairy products; excessive stress are causal in severe acneiform eruption $[4,5]$. Psychological stress release stress hormones like Tumour Necrosis Factor(TNF) from CNS. This with an increase consumption of bovine milk products change the enteric microbiota of the lining of the gastrointestinal tract. A lack of high fibre in the diet slows the gut motility. Toxins are produced by the dysbiosis microbiota of the small gut. The leaky epithelium become more permeable and the toxins are readily absorbed. The toxaemia promotes secretions of Substance $\mathrm{P}$ and other stress hormones like IGF, corticosteroids and cytokines mediators especially tumour necrosis factors systemically [6]. Active inflammation of the pilosebaceous unit of the skin dermis while uncontrolled secreted high saturated fatty acid contents seborrhoea, hyperkeratinisation of the corneocytes and proliferation of Propionibacterium acne (P. acne). A pathological ferocious cycle act on genetically susceptible individuals resulted severe scar prone acne. Insulin resistance at the cellular level has been demonstrated in these severe acne susceptible individuals. Recent study involving breath test methodology showed that $\mathrm{P}$. acne may adhere to intestinal mucosa [7]. Systemic isotretinoin may induce mucosal side effects with signs of altered immunity. Patients with severe acne treated with oral systemic isotretinoin was shown to present with small gut dysbiosis identified by breath test [7]. These patients presented with frequent relapses of oral Herpes simplex infections or allergy [7]. Systemic isotretinoin which has been used successfully for decades for managing severe acne may aggravate IBD especially ulcerative colitis $[7,8]$.

In Rosacea, a more complex interaction between the brain, nervous system, cardiovascular system, the gut and the skin take place. The prevalence of celiac disease, Crohn's disease, ulcerative colitis, Helicobacter pylori (H. pylori) infection, irritable bowel and small intestinal bacterial overgrowth were all higher among Rosacea patients than the study controls [9-11]. Stressors of Rosacea like spices, stress and heat act through the widely distributed Transient Receptor Potential (TRP) channels receptors family; TRPV1, TRPV2, TRPV3, TRPV4, TRPA1 and TRPM located on neuronal endings sensitize the autonomic nervous system both the sympathetic and parasympathetic pathways causing vasodilatation like flushing and hyperalgesia stinging sensation [12,13]. Nerves containing Calcitonin genes related peptide (CGRP) may be 


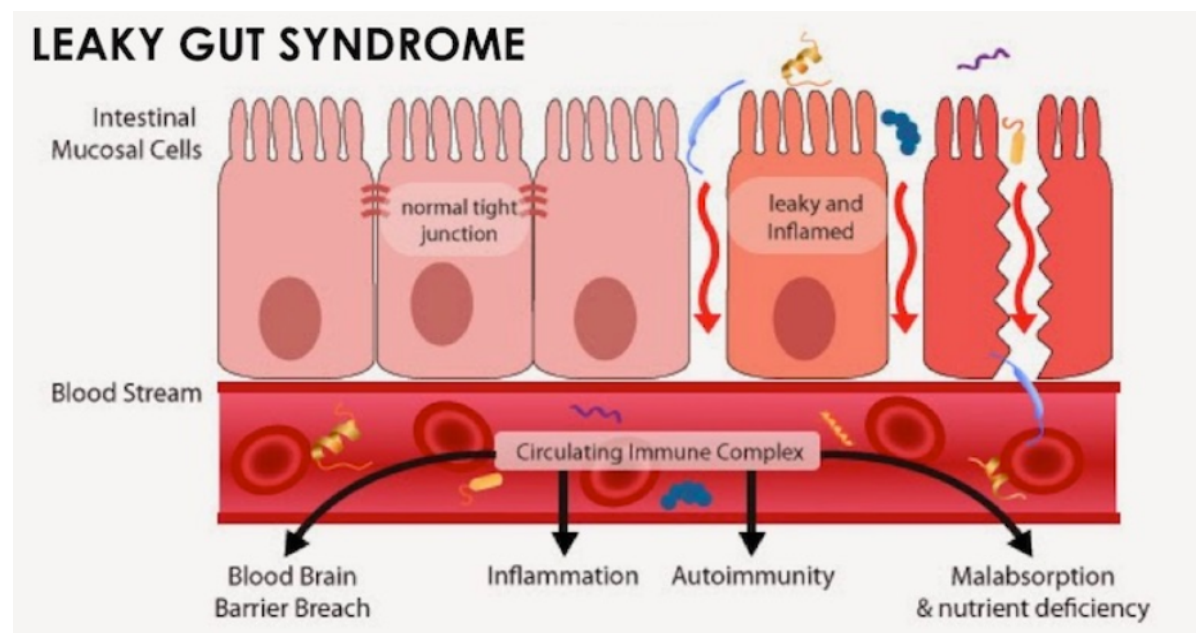

Figure 1. Leaky gut. (Source: https://juicing-for-health.com/how-to-heal-leaky-gut).

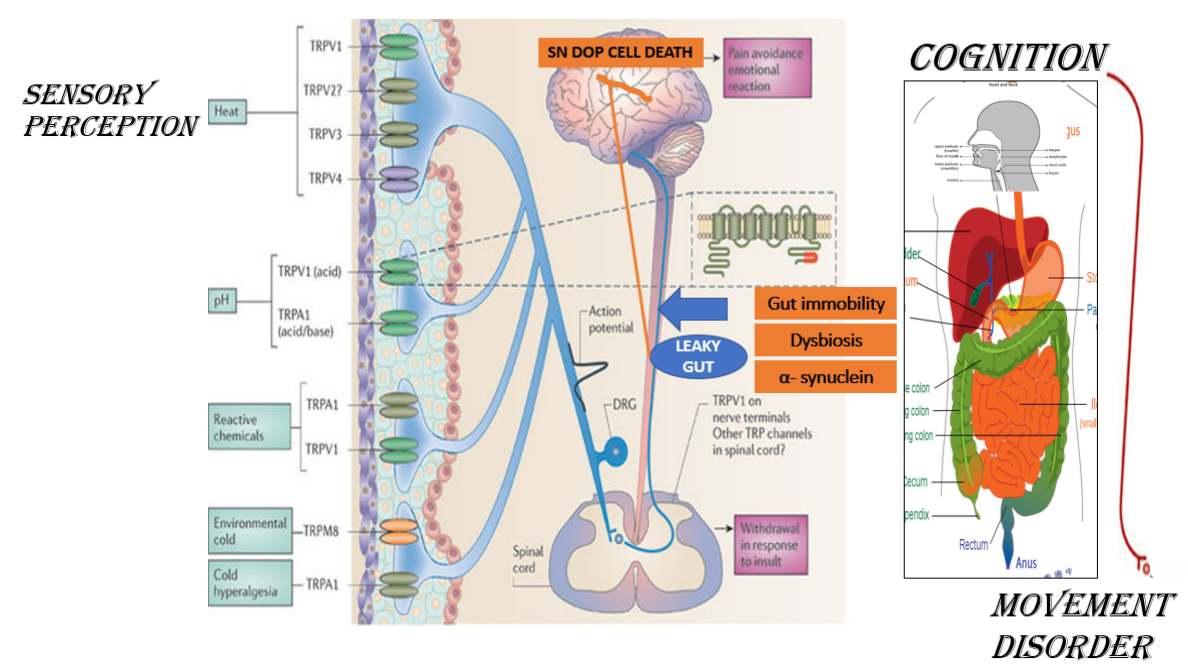

Figure 2. Adapted from Nature Review (SN DOP=Substantial nigra Dopaminergic neuronal cells deaths due to $\alpha$ - synuclein).

linked to flushing through the messenger cAMP. TRPV act like a N-methyl-D-aspartate (NMDA) receptor cause calcium influx thus changes transmembrane potential transmits signals to the cardiovascular system via the CNS. The angiogenesis and inflammatory insults cause a direct neurogenic vascular effect on the facial sensitive dry skin resulting erythema, telangiectasia, papules and pustules; pathognomonic phenotypic manifestations of Rosacea [14]. Epidermal barrier defects and body self - defence to pathogenetic bacterium in the skin epidermis and intestinal mucosa with the production of potent antibacterial cathelicidin (LL37); produced through the Kallikrein 5 pathway involving the Matrix metalloproteinases (MMP). LL-37 has been demonstrated to be a major pathogenetic factor in causing rosacea through inflammation and angiogenesis of skin [15-18] Auto - antibodies has been detected against $H$. pylori in Rosacea give evidence that stasis, reflux, an acidic $\mathrm{pH}$ environment of the small intestine favour proliferation, invasion and pathogenicity of $\mathrm{H}$. pylori [19]. Systemic antibiotics including the macrolide group is efficacious in managing Rosacea clinically especially the papulopustular subtypes [20]. A reduction of gut transit time using a high-fibre intervention resulted in improvement of Rosacea. Other enteric bacterium in the small gut including the oral cavity may also be involved and should be investigated.

\section{Atopic dermatitis and probiotics}

Leaky skin behaves similarly as the leaky gut; the outside world invades but inside world drain and get exhausted. Emerging evidence suggested that if there is a better control and management of the innate defective skin; i.e.; minimizing Trans Epidermal Water Loss (TEWL), desensitisation of allergens and maintenance of a healthy microbial flora over the skin surface, associated $\mathrm{AD}$ diseases like food allergies, rhinitis, hay fever, asthma and irritable bowel may be reduced in incidence [21]. Food allergies is highly associated with AD and up to one third of patients with skin allergy has inflammation of gut. Synbiotics defined as probiotics in combination with prebiotics have been shown to be beneficial in the management of $\mathrm{AD}$. The former is living cultures while the latter is indigestible polysaccharide which can stimulate the growth of the former. The mind with all its cognitive domains have an effect on AD. Sleep disturbances, emotional disorders like depression and compulsive disorders are main cognitive players; though not exclusive; in severe $\mathrm{AD}$ especially during the kicking off of the itch-scratch cycle $[22,23]$. Recognition and understanding of the Brain-GutEnteric microbiota - Skin axis interplay is essential to managing doctors in managing the AD children or adults and their families. This is a crucial step to enable the best management of chronic recalcitrant $\mathrm{AD}$ either in children or adults. Liberal 
applications of moisturizers, use of appropriate cleansers, life style adjustments, avoidance of triggers, balanced diet with avoidance of processed foods, cognitive behaviour therapy, relaxation, meditation and state of the art medications should all be explained and discussed in details; especially the pros and cons; to the individual patients $[23,24]$. Personalized treatment strategy involving the patients and concerned family members should be worked out and planned in advance and proactively before flare during remission to achieve the least distress and sufferings of the chronic $\mathrm{AD}$ patients [23].

\section{Parkinson Disease, Gut and the skin}

The aetiology of PD is multifactorial and complex but also illustrated the CNS-Gut-Skin axis relationship [25-29]. Genetic predictions like R1441G mutation, LRRK2 mutation, Parkin and PINK 1 mitogenic mutation and the deleterious effects of 1-methyl-4-phenyl-1,2,3,6-tetrahydropyridine (MPTP) causing mitochondrial dysfunction are known causations [25]. Recently the accumulation of a neuronal protein transfers from the gut, glial cells and enteric nervous system (ENS) deposited in the substantia nigra (SN) of the basal ganglion of the brain cause gradual dopaminergic neuronal cell deaths through apoptosis and inflammation is under active research [30-32]. There is a gradual depigmentation of the SN in PD compared with the studied controls due to the loss of neuromelanin. Non- Motor Parkinsonism (NMP) manifestations are diverse but include the GI tract and the skin. Gut immobility, constipation with the subsequent bacterial overgrowths and dysbiosis of the gut lining favour absorption of toxins. $\mathrm{H}$ pylori may play a role in PD. The neuronal protein under intense study is called $\alpha$ synuclein. $\alpha$-synuclein is a neurotoxic protein when accumulate can cause cell deaths with the formation of Lewy Bodies found in the dopaminergic neurone in the brain in PD. Lewy Bodies are pathognomonic of PD in histopathology. $\alpha$ - synuclein and Lewy Bodies are found widely distributed in the ENS of the gut, olfactory bulbs, sympathetic and parasympathetic ganglions, spinal cords, neuronal cells of the brain and the skin. The gut microbiota interaction with the enteric neurons, enteric glial cells as counterpart of the brain astrocytes seem to be involved due to the dysfunction of GI tract and develop PD. Much like food allergies and $\mathrm{AD}, \mathrm{PD}$ patients neuro chemical changes with production of inflammatory cytokines with impairment of intestinal epithelia barrier has been observed. There are also dysregulation and loss of enteric glial cells with $\alpha$ - synuclein formation. A positive correlation between gut permeability and deregulation of enteric glial cells and intestinal $\alpha$ - synuclein have been confirmed in studies. $\alpha$ - synuclein plays a crucial role in neuro-inflammation by triggering on/off potentiating glial cell activity. Caffeine ingestion and cigarette smoking reduce small gut inflammation leads to less aggregation of Synuclein in the ENS, known to decrease the risk of PD. A rostrocaudal gradient of synucleinopathy within the GI tract with submandibular gland and lower oesophagus having the highest density of Lewy Bodies followed by the stomach, small intestine, colon and rectum [25]. This rostrocaudal distribution of Lewy pathology coincides with vagal innervation from dorsal motor nucleus of the vagus (DMNV) as well as the distribution of dopaminergic neurones in the ENS [25]. Furthermore, peripheral induced inflammation can induce central inflammation and result dopaminergic pathway degeneration.

Apart from the gut, nonmotor manifestations of PD also involve the oral cavity with diseases like periodontal diseases, poor oral hygiene, dental problems with loss of teeth and bad breath [33]. A Dual hit theory has been postulated that neurodegenerative process starts in the olfactory bulbs and ENS of the gut after following inhalation and ingestion of a neurotropic infectious proteinaceous agent [34]. A possible candidate is the prion protein. The neuropathological process that leads to PD seems to start in the upper part of the gut with synucleinopathy throughout the whole GI tract. The ENS, the gut microbiota dysbiosis participate and enhance the active transport of $\alpha$ synuclein via the vagal nerve to the CNS [35,36]. Maintaining the balance of microbiota of the gut and its environment may provide a beneficial effect to prevent the development of PD; mimicking the scenario of synbiotics prevention in children developing AD. Dietary advises with appropriate probiotics and prebiotics administration at relevant time and dosage may be a cost-effective solution in the prevention of PD [37].

Early stage of PD is easily recognised from the skin [38]. There are many skin manifestations of PD like seborrhoea in post encephalitic PD. Severe seborrhoea and scarring acne is referred by some as pre-motor feature of PD. There is a frequent overlap between patient presented with seborrheic dermatitis and PD. The pathological Malassezia yeasts especially the Malassezie globosa has a higher density in PD skin. Hyperhidrosis is not uncommon in PD patients particularly after taking Levo-Dopa. Flushing due to vascular instability and bullous pemphigoid due to autoantibodies formation to epidermal barrier are reported skin associations. The skin of PD patients should be carefully examined and documented for evidence of early sign of melanoma as $\alpha$-synuclein is implicated in the pathogenesis of the latter [39,40]. Testing of $\alpha$ - synuclein in skin histology through small punch diagnostic skin biopsy is a safe, minimally invasive clinic base procedure which has been shown and should be further scientifically studied as a cost- effective screening strategy of early NMP $[41,42]$.

\section{Conclusion}

The physiological interaction of the most superficial layer of the body: skin and the most inner organ: the gut through the peripheral and central nervous system maintain homeostasis, defence, sensory, absorptive and cognitive functions of our body enable us to survive and remain healthy in an unstable ever changing ecological surroundings. Environmental factors including pathogens, pollutants, dietary habits and genetic predisposition result from bad gene mutations interact with dysbiosis of the gut perverted microbiota generate clinically seemingly unrelated diseases. The GI tract especially its motility and dysbiosis, CNS with its cognitive and movement domains, peripheral nervous system and skin should be integrally studied in many previously chronic, difficult- to- treat diseases. In this short review, Acneiform facial skin eruption, Chronic Atopic Dermatitis, Parkinson Disease are revisited and discussed under this perspective. Understanding the physiological interplay of these organs and its subsequent pathogenic state upon viscous 
insults enlighten us how internally the body system works integrally. This may provide us new insight how nature works and therapeutically; better management strategy for these distressing conditions.

\section{Acknowledgement}

Part of the article materials are extracted from discussions during the conference sessions of the Proceedings of the 17th European Dermatology Congress held in Paris, France; 1-3 March.

Special thanks to Professor Donatini Bruno, Medicine Information Formation, France and Professor Lazaros I Sakkas, University of Thessaly, Greece.

\section{References}

1. Chan KTM. Severe Acneiform Facial Eruption: An Updated Prevention, Pathogenesis and Management. Med Clin Res; 2017;2(2):1-55.

2. Williams HC, Dellavalle RP, Garner S. Acne vulgaris. Lancet 2012;361-72.

3. Whitney P Bowe, Logan AC. Acne vulgaris, probiotics and the gut-brain-skin axis - back to the future? Gut Pathogens 2011;3:1.

4. Smith TM, Cong Z, Gilliland KL, et al. Insulin-like growth factor-1 induces lipid production in human SEB-1 sebocytes via sterol response element-binding protein-1. J Invest Dermatol 2006;1226-32.

5. Smith RN, Mann NJ, Braue A, et al. The effect of a highprotein, low glycemic-load diet versus a conventional, high glycemic-load diet on biochemical parameters associated with acne vulgaris: a randomized, investigator-masked, controlled trial. J Am Acad Dermatol 2007;57:247-56.

6. Zouboulis CC, Böhm M. Neuroendocrine regulation of sebocytes - a pathogenetic link between stress and acne. Exp Dermatol 2004;13:31-5.

7. Bruno D. Severe acne treated with isotretinoin in associated with dysbiosis and its consequences. J Clin Exp Dermatol Res 2018;9.

8. Crockett SD, Porter CQ, Martin CF, et al. Isotretinoin use and the risk of inflammatory bowel disease: a casecontrol study. The American Journal of Gastroenterology. 2010;105(9):1986-93.

9. Kyriakis KP, Palamaras I, Terzoudi S, et al. Epidemiologic aspects of rosacea. JAAD 2005;53:918-9.

10. Rainer BM, Fischer AH, Luz Felipe da Silva D, et al. Rosacea is associated with chronic systemic diseases in a skin severity-dependent manner: results of a case-control study. J Am Acad Dermato 2015;73:604-8.

11. Spoendlin J, Karatas G, Furlano RI, et al. Rosacea in patients with ulcerative colitis and Crohn's disease: a population-based case-control study. Inflammatory Bowel Disease 2016;22:680-7.

12. Two AM, Wu W, Gallo RL, et al. Rosacea: part I.
Introduction, categorization, histology, pathogenesis, and risk factors. Pathways known to contribute to the pathophysiology and clinical manifestations of Rosacea. JAAD 2015;72:749-58.

13. Sulk M, Seeliger S, Aubert J, et al. Distribution and expression of non-neuronal transient receptor potential (TRPV) ion channels in rosacea. Journal of Investigative Dermatology 2011;132:1253-62.

14. Helfrich YR, Maier LE, Cui Y, et al. J. Clinical, histologic, and molecular analysis of differences between erythematotelangiectatic rosacea and telangiectatic photoaging. JAMA Dermatol 2015;151:825-36.

15. Schwab VD, Sulk M, Seeliger S, et al. Neurovascular and neuroimmune aspects in the pathophysiology of rosacea. Journal of Investigative Dermatology Symposium Proceedings 2011;15:53-62.

16. Zhou M, Xie H, Cheng L, et al. Clinical characteristics and epidermal barrier function of papulopustular rosacea: a comparison study with acne vulgaris. Pak J Med Sci 2016;32:1344-8.

17. Yamasaki K, DiNardo A, Bardan A, et al. Increased serine protease activity and cathelicidin promotes skin inflammation in rosacea. Nature Medicine 2007;13:975-80.

18. Reinholz M, Ruzicka T, Schauber J. Cathelicidin LL- 37; an antimicrobial peptide with a role in inflammatory skin disease. Ann Dermatol 2012;24:126 -35.

19. Diaz C, O'Callaghan CJ, Khan A, et al: Rosacea: a cutaneous marker of Helicobacter pylori infection? Acta DermatoVenereologica. 2003; 83:282-6

20. Utas S, Ozbakir O, Turasan A, et al. Helicobacter pylori eradication treatment reduces the severity of rosacea. Journal of the American Academy of Dermatology. 1999;40:433-5.

21. Cork M. J Allergy Clin Immunol 2006;118:3-21.

22. Chan KTM. Der Pharmacia Lettre, 2018;10[3]:1-2.

23. Sanders KM, Nattkemper LA, Yosipovitch G. Advances in understanding itching and scratching: A new era of targeted treatments 2016 .

24. Zirwas MJ. The Future is Finally Here: Advances in the Treatment of Atopic Dermatitis, Journal of the American Academy of Dermatology 2018.

25. Klingelhoefer L, Reichmann H. The Gut and Nonmotor Symptoms in Parkinson's Disease. International Review of Neurobiology. 2017;134:788-800.

26. Klingelhoefer L, Reichmann H. Pathogenesis of Parkinson disease-The gut brain axis and environmental factors. Nature Reviews. Neurology, 2015;11(11):625-36.

27. Dinan TG, Cryan, JF. Gut instincts: Microbiota as a key regulator of brain development, ageing and neurodegeneration. The Journal of Physiology. 2017;595(2):489-503. 
28. Mayer EA, Tillisch K, Gupta A. Gut/brain axis and the microbiota. The Journal of Clinical Investigation. 2015;125(3):926-38.

29. Cryan JF, Dinan TG. Mind-altering microorganisms: The impact of the gut microbiota on brain and behaviour. Nature Reviews. Neuroscience, 2012;13(10):701-12.

30. Gao HM, Zhang F, Zhou H, et al. Neuroinflammation and alpha-synuclein dysfunction potentiate each other, driving chronic progression of neurodegeneration in a mouse model of Parkinson's disease. Environmental Health Perspectives, 2011;119(6):807-14.

31. Stokholm MG, Danielsen EH, Hamilton-Dutoit SJ, et al. Pathological alpha-synuclein in gastrointestinal tissues from prodromal Parkinson disease patients. Annals of Neurology. 2016;79(6):940-9.

32. Sanchez-Guajardo V, Tentillier N, Romero-Ramos M. The relation between alpha-synuclein and microglia in Parkinson's disease: Recent developments. Neuroscience. 2015;302:47-58.

33. Bakke M, Larsen SL, Lautrup C, et al. Orofacial function and oral health in patients with Parkinson's disease. European Journal of Oral Sciences. 2011;119(1):27-32.

34. Reichmann H. View point: Etiology in Parkinson's disease. Dual hit or spreading intoxication. Journal of the Neurological Sciences, 2011;310(1-2):9-11.

35. Ghaisas S, Maher J, Kanthasamy A. Gut microbiome in health and disease: Linking the microbiome-gut-brain axis and environmental factors in the pathogenesis of systemic and neurodegenerative diseases. Pharmacology \& Therapeutics. 2016;158:52-62.

36. Sampson TR, Debelius JW, Thron T, et al. Gut microbiota regulates motor deficits and neuroinflammation in a model of Parkinson's disease. Cell. 2016;167(6):1469-80.

37. Voreades N, Kozil A, Weir TL. Diet and the development of the human intestinal microbiome. Frontiers in Microbiology. 2014;5:494.

38. Arsenijevic V, Milobratovic D, Barac A, et al. A laboratory based study on patients with Parkinson's disease and seborrhoeic dermatitis. BMC Dermatol 2014;14:5.

39. Pan T, Zhu J, Hwu W, et al. The Role of Alpha-Synuclein in Melanin Synthesis in Melanoma and Dopaminergic Neuronal Cells. PLOS ONE 2012;7:451-83.

40. Bertoni J, Arlette J, Fernandez H, et al. Increased Melanoma Risk in Parkinson Disease: A Prospective Clinicopathological Study. Arch Neurol. 2010;67:347-52.

41. Malek N, Swallow D, Grosset KA, et al. Alpha-synuclein in peripheral tissues and body fluids as a biomarker for Parkinson's disease-a systematic review. Acta Neurol Scand. 2014;130:59-72.

42. Schneider SA, Boettner M, Alexoudi A, et al. Can we use peripheral tissue biopsies to diagnose Parkinson's disease? A review of the literature. European Journal of Neurology. 2016 23(2):247-61.

\section{*Correspondence to:}

Chan KTM

Specialist in Dermatology

Hong Kong Academy of Medicine

Hong Kong

Tel+852-21282129

E-mail: pioneerskin@ymail.com 